\title{
Gerenciamento de áreas contaminadas por postos de serviços em Goiânia/GO
}

Terrenos quimicamente degradados por derivados de petróleo constituem o maior número de áreas contaminadas no mundo. Apesar dos riscos ambientais e humanos potenciais associados aos hidrocarbonetos, pesquisas voltadas ao gerenciamento de passivos ambientais desencadeados por postos de serviços ainda são raras no Brasil. O presente trabalho foi desenvolvido no período de agosto/2017 a julho/2018, mediante a análise dos estudos ambientais constantes em 200 processos de licenciamento de postos de serviços existentes na Agência Municipal do Meio Ambiente de Goiânia, Goiás, Brasil. Após a aplicação da Metodologia GAC, cada estabelecimento foi enquadrado em uma das categorias de gerenciamento de áreas contaminadas estabelecidas pela Resolução no 420/2009, do CONAMA. Do total de processos analisados, $22 \%$ dos empreendimentos foram classificados como Áreas Suspeitas de Contaminação, $11,5 \%$ como Áreas Contaminadas sob Investigação, 7,5\% como Áreas Contaminadas sob Intervenção, 1,5\% como Áreas em Processo de Monitoramento para Reabilitação e 57,5\% como Áreas Livres de Contaminação. A espacialização dos resultados possibilitou identificar quatro aglomerados de terrenos demandantes de ações gerenciais prioritárias por parte da municipalidade, dados os riscos de exposição ambiental e humana às substâncias químicas de interesse. Considerando a contínua expansão da rede de postos de serviços em Goiânia e nos demais núcleos urbanos do país, bem como os desafios de gestão associados aos passivos ambientais oriundos do setor, fica evidente a necessidade de aplicação de medidas gerenciais sistêmicas visando, acima de tudo, a reintegração ao tecido urbano dos terrenos impactados.

Palavras-chave: Passivo ambiental; Hidrocarbonetos; Sustentabilidade urbana.

\section{Management of contaminated areas by service stations in Goiânia/GO}

\begin{abstract}
The largest number of contaminated areas in the world are land chemically degraded by petroleum products. Despite the potential environmental and human risk associated with hydrocarbons, researches into the management of environmental liabilities triggered by fuel stations in Brazil are still rare. This work was developed in the period from August/2017 to July/2018, through the analysis of the environmental studies included in 200 licensing processes for fuel stations in the local Environment Agency of Goiânia, Goiás, Brazil. After applying the GAC Methodology, each establishment was categorized according to Resolution no $420 / 2009$ of CONAMA for the management of contaminated areas. After analyzing all the processes, $22 \%$ of the projects were classified as Suspicious Areas of Contamination, $11.5 \%$ as Polluted Areas under Investigation, $7.5 \%$ as Polluted Areas under Intervention and $1.5 \%$ as Pollution Free Areas. The spatialization of the results made it possible to identify four clusters of land requiring priority management actions by the local government, given the risks of human and environmental exposure to the chemicals of interest. Considering the continuous expansion of the network of fuel stations in Goiânia and in the other urban centers of the country and the environmental management challenges associated with the liabilities arising from the sector Considering the continuous expansion of the network of fuel stations in Goiânia and other urban centers of the country and the environmental management challenges associated with the liabilities arising from the sector, it is evident the need to apply systemic managerial decisions aimed above all at the reintegration to the urban set of impacted terrains.
\end{abstract}

Keywords: Environmental liabilitie; Hydrocarbons; Urban sustainability

Topic: Engenharia Ambiental

Reviewed anonymously in the process of blind peer.
Received: 04/05/2020

Approved: 01/07/2020
Alecrícia Barros Silva in

Instituto Federal de Goiás, Brasil

http://lattes.cnpq.br/0383079163913960

http://orcid.org/0000-0002-9163-453X

alecriciabarross@gmail.com

Francislainy Teles Almeida Valverde

Instituto Federal de Goiás, Brasil

http://lattes.cnpq.br/6542824490178970

francisteles@hotmail.com

Guilherme Matheus Coelho de Lemos

Instituto Federal de Goiás, Brasil

http://lattes.cnpq.br/8436251789474385

http://orcid.org/0000-0003-3822-9065

guilhermemcoelhoo@gmail.com

\author{
João Messias Fernandes \\ Instituto Federal de Goiás, Brasil \\ http://lattes.cnpq.br/4496509506236216 \\ joaomessiasambiental@gmail.com \\ Rosana Gonçalves Barros \\ Instituto Federal de Goiás, Brasil \\ http://lattes.cnpq.br/3009142457782923 \\ http://orcid.org/0000-0003-4007-3450 \\ rosana.ifg@gmail.com \\ Viníciu Fagundes Bárbara (iD \\ Instituto Federal de Goiás, Brasil \\ http://lattes.cnpq.br/3052075493147161 \\ http://orcid.org/0000-0001-9243-5543 \\ viniciu.fagundes@ifg.edu.br
}

\section{Referencing this:}

SILVA, A. B.; VALVERDE, F. T. A.; LEMOS, G. M. C.; FERNANDES, J. M.; BARROS, R. G.; BÁRBARA, V. F.. Gerenciamento de áreas

contaminadas por postos de serviços em Goiânia/GO. Nature and Conservation, v.13, n.3, p.66-79, 2020. DOI:

http://doi.org/10.6008/CBPC2318-2881.2020.003.0007 


\section{INTRODUÇÃO}

Áreas contaminadas, também denominadas de passivos ambientais, se constituem em terrenos alterados por substâncias químicas capazes de degradar matrizes como solo, águas superficiais e subterrâneas e sedimentos, bem como de causar danos aos seres vivos (SAM et al., 2017; ISTRATE et al., 2018). Em diversos países são encontrados sites contaminados oriundos de disposições finais ambientalmente inadequadas de resíduos sólidos advindos de indústrias como curtumes, mineradoras e fábricas de baterias, e de áreas de manufatura e de aplicação de agrotóxicos (BOCOS et al., 2015; RODRÍGUEZ et al., 2018). Apenas na Europa existem cerca de 2,5 milhões de terrenos quimicamente degradados atualmente, dos quais 350.000 são considerados de elevada periculosidade (VALENTYNE et al., 2018).

Ao serem introduzidos no meio ambiente e dependendo de suas características físico-químicas, os diferentes tipos de contaminantes perigosos utilizados rotineiramente nas atividades humanas tendem a ser transportados pelas correntes atmosféricas ou águas superficiais e subterrâneas, podendo resultar em impactos ambientais de difícil mitigação (BALKIS et al., 2012; BALSEIRO-ROMERO et al., 2016). Hidrocarbonetos como benzeno, tolueno, etilbenzeno e xilenos (BTEXs) se destacam nesse contexto, pois apresentam elevado potencial toxicológico, são mais solúveis no meio aquático e são demandados em altas quantidades pela sociedade, notadamente na forma de combustíveis (SINGH et al., 2014; CAMENZULI et al., 2015; ZELINKOVA et al., 2015).

Segundo Brow et al. (2004), Djomo et al. (2004) e Chen et al. (2011), hidrocarbonetos são substâncias presentes na lista de poluentes prioritários da União Europeia e dos Estados Unidos da América (EUA) há décadas, dado seu potencial de degradação ambiental e humano. Especificamente, se constituem em elementos capazes de desencadear mutações e enfermidades mediante a absorção via tecidos, ingestão direta de água ou de alimentos contaminados e inalação de vapores. Souza et al. (2014) destacam que o tolueno, o etilbenzeno e os xilenos apresentam toxicidade sistêmica de baixa a moderada, podendo comprometer o sistema nervoso central de organismos expostos, além de serem embriotóxicos e fetotóxicos. O benzeno, reconhecidamente o mais tóxico, é cancerígeno e mutagênico (UGOCHUKWU et al., 2018). Nesse sentido, Khan et al. (1996) pesquisaram os efeitos bioquímicos clínicos e sistêmicos à saúde de bovinos expostos a hidrocarbonetos em Obuaki, Nigéria, tendo comprovado riscos significativos à integridade física dos animais, incluindo tremores, vômito e angústia pulmonar, além de alterações em rins, fígado e pulmões. De acordo com os autores, efeitos semelhantes já foram observados em outros mamíferos, inclusive no homem.

Atualmente, a contaminação ambiental por hidrocarbonetos advindos de vazamentos em Sistemas de Armazenamento Subterrâneos de Combustíveis (SASCs) de postos de serviços é uma das principais preocupações dos órgãos ambientais (ROSALES et al., 2014), pois o setor responde pelo maior número de áreas contaminadas no mundo (TEIXEIRA et al., 2016), principalmente em países como Alemanha, Inglaterra, Japão, EUA e Canadá. Neste último, por exemplo, as perdas de combustíveis em 2009 totalizaram 58.300.000 $\mathrm{L}$, resultando em uma estimativa de, aproximadamente, 1,7 $\mathrm{L}$ de gasolina liberada per capita por ano 
(HILPERT et al., 2015; ADIPAH, 2019).

Nacionalmente, contaminações por hidrocarbonetos ocorrem em todos os Estados. Em São Paulo (SP), por exemplo, estabelecimentos que trabalham com combustíveis estão associados a cerca de $71 \%$ dos passivos ambientais registrados, enquanto que em Minas Gerais (MG) e no Rio de Janeiro (RJ), a 77\% e 56\%, respectivamente (ROSALES et al., 2014). No estudo desenvolvido por Lima et al. (2017) em Cuiabá, Mato Grosso (MT), foi comprovado que no universo de 136 postos pesquisados, pelo menos 17 apresentavam algum tipo de contaminação por BTEXs, oferecendo riscos potenciais à população exposta. Em Rondônia, um terreno localizado em Porto Velho apresentou contaminação em diversas amostras de águas subterrâneas, inclusive de poços utilizados como fontes de abastecimento (FORTE et al., 2007). Resultados semelhantes foram obtidos por Vasconcelos et al. (2014), em trabalho desenvolvido em São Bernardo do Campo (SP); Silva et al. (2002), em pesquisa realizada em Itaguaí (Rio de Janeiro, RJ), e Dias (2012), em Natal, Rio Grande do Norte (RN). Considerando que o Brasil conta com cerca de 40.000 postos em operação, o potencial de contaminação associado ao setor se mostra elevado e demandante de ações gerenciais específicas na busca pela sustentabilidade (ITE et al., 2018), as quais devem passar pelo desenvolvimento de diagnósticos capazes de caracterizar adequadamente os riscos ambientais, sociais e econômicos associados às áreas contaminadas, inclusive com vistas à otimização dos recursos financeiros destinados para fins de recuperação (FREIRE et al., 2014).

Um dos principais agravantes da problemática das áreas contaminadas por hidrocarbonetos no Brasil é que os atuais instrumentos regulatórios ainda são poucos e fragmentados. Nesse sentido, somente em 2000, com o advento da Resolução no 273/2000 (BRASIL, 2000), do Conselho Nacional de Meio Ambiente (CONAMA), passou a ser obrigatória a elaboração de Investigações de Passivos Ambientais (IPAs) para fins de licenciamento de postos de serviços. Posteriormente, a Resolução no 420/2009, também do CONAMA, estabeleceu os critérios e valores orientadores de qualidade do solo quanto à presença de substâncias químicas, bem como definiu as diretrizes mínimas para o gerenciamento de áreas contaminadas em decorrência de atividades antrópicas. Diante desses marcos regulatórios, os órgãos licenciadores passaram a estabelecer metodologias específicas de identificação, diagnóstico e intervenção voltadas para a gestão de estabelecimentos potencialmente desencadeadores de contaminações, como postos de serviços.

Especificamente, para a garantia da qualidade ambiental e a prevenção de contaminações em terrenos ocupados por estabelecimentos comercializadores de combustíveis, a Resolução no 273/2000 do CONAMA estabeleceu a obrigatoriedade de serem implantados programas de monitoramento periódico da qualidade do solo e das águas subterrâneas, cujos resultados devem ser descritos em relatórios técnicos conclusivos apresentados a cada solicitação de renovação de licença ambiental, ou antes do encerramento das atividades operacionais (BRASIL, 2009). Dessa forma, o poder púbico passou a ter conhecimento prévio da existência de sites contaminados por hidrocarbonetos e a exigir a adoção de medidas de recuperação. Entretanto, apesar da instituição de marcos regulatórios e metodológicos, a grande maioria das informações oriundas dos estudos ambientais investigativos desenvolvidos em postos de combustíveis ainda não necessariamente resulta em medidas eficientes de gerenciamento por parte dos órgãos licenciadores 
(ARAÚJO-MOURA et al., 2015), dado o elevado nível de desconhecimento sobre o gerenciamento de áreas contaminadas no país.

Embora Goiânia (Goiás, GO) seja uma das capitais brasileiras com a maior quantidade de postos de serviços em operação, ainda existem poucas informações científicas sobre os passivos ambientais advindos do setor, o que resulta em medidas gerenciais pouco eficientes por parte do poder público. Portanto, o objetivo do presente estudo foi aplicar a Metodologia de Gerenciamento de Áreas Contaminadas (Metodologia GAC) em postos de serviços a fim de traçar um panorama para fins de gerenciamento ambiental prioritário em Goiânia.

\section{METODOLOGIA}

O estudo foi desenvolvido em Goiânia, capital do Estado de Goiás, Brasil, situada na Mesorregião Centro-Goiano (Figura 1). Ocupando uma área de $728.841 \mathrm{~km}^{2}$ e com 1.448 .639 habitantes (IBGE, 2016), o município conta com clima tropical, temperatura média anual de $21,3 \circ \mathrm{C}$ e topografia predominantemente plana. A geologia local é formada por rochas metamórficas do Grupo Araxá, caracterizada por xistos, quartzitos, granada, feldspatos e concentrações variáveis de micas (SGM, 2005).

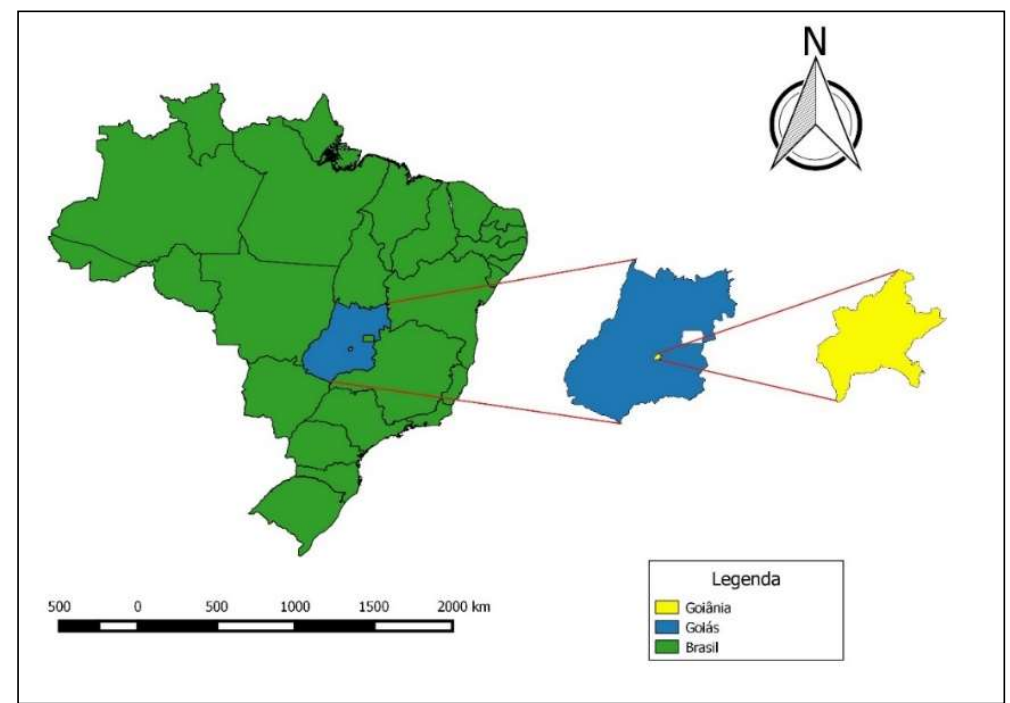

Figura 1: Localização do município de Goiânia em relação ao Estado de Goiás e ao Brasil.

A Agência Municipal do Meio Ambiente de Goiânia (AMMA) é uma autarquia com autonomia administrativa, financeira e patrimonial cuja responsabilidade consiste em formular, implementar e coordenar a execução da Política Municipal do Meio Ambiente (PMMA). No período de agosto/2017 a julho/2018, seu Departamento de Licenciamento Ambiental disponibilizou os estudos técnicos - Relatório de Investigação de Passivo Ambiental Preliminar (RIPA Preliminar); Relatório de Investigação de Passivo Ambiental Confirmatória (RIPA Confirmatória); Relatório de Investigação de Passivo Ambiental Detalhada (RIPA Detalhada); Relatório de Análise de Risco (RAR) e Relatório de Monitoramento Preventivo (RMP) - mais recentes (Metodologia GAC) (BRASIL, 2009; CETESB, 2017), integrantes dos processos de licenciamento de 200 dos postos de serviços em funcionamento no município, os quais foram sistematicamente analisados pelos autores para a obtenção de dados da qualidade das águas subterrâneas e de solo de cada terreno. 
A Metodologia GAC é dividida em seis etapas sequenciais: (i) Investigação de Passivo Ambiental Preliminar (IPA Preliminar); (ii) Investigação de Passivo Ambiental Confirmatória (IPA Confirmatória); (iii) Investigação de Passivo Ambiental Detalhada (IPA Detalhada); (iv) Análise de Risco (AR); (v) Plano de Intervenção (PI) e (vi) Monitoramento Preventivo (MP). A IPA Preliminar objetiva identificar a existência de indícios de contaminação ou de condições ambientalmente perigosas, tendo por base informações do histórico e das instalações do estabelecimento. Nessa etapa também são realizadas medições de compostos orgânicos voláteis (COVs) em diferentes profundidades no solo. Se identificadas situações suspeitas ou quantificados gases de petróleo na subsuperfície, os levantamentos devem seguir para a IPA Confirmatória, que busca comprovar a existência de hidrocarbonetos nas formas líquida (fase livre), sorvida e/ou dissolvida acima dos valores máximos estabelecidos por Brasil (2009). Em caso positivo, parte-se para a realização da IPA Detalhada, que deve apresentar a delimitação integral das plumas de contaminantes, em termos de área e volume, de forma a promover o adequado entendimento da dinâmica da contaminação, dos cenários específicos de uso e ocupação do solo, dos receptores de risco, dos caminhos de exposição e das vias de ingresso, culminando com o detalhamento do modelo conceitual do site. Na sequência, é realizada a Análise de Riscos, que implicará na elaboração do Plano de Intervenção (PI) ou, em inexistindo riscos significativos à saúde humana ou aos bens de relevante interesse ambiental a proteger, no Monitoramento Preventivo.

Quadro 1: Categorias de enquadramento estabelecidas por Brasil (2009), com adaptações, para fins de gerenciamento de áreas contaminadas em Goiânia/GO.

\begin{tabular}{|c|c|}
\hline Categoria & Descrição \\
\hline $\begin{array}{l}\text { Área } \quad \text { Suspeita } \\
\text { Contaminação (AS) }\end{array}$ & $\begin{array}{l}\text { Aquela onde, após a realização de IPA Preliminar, foram identificados indícios da presença de } \\
\text { contaminação ou condições que possam representar perigo. }\end{array}$ \\
\hline $\begin{array}{l}\text { Área Contaminada sob } \\
\text { Investigação (Al) }\end{array}$ & $\begin{array}{l}\text { Área onde comprovadamente foi constatada, mediante IPA Confirmatória, a concentração de } \\
\text { substâncias no solo e/ou nas águas subterrâneas acima dos valores máximos estabelecidos pela } \\
\text { Resolução no } 420 / 2009 \text {, do CONAMA. }\end{array}$ \\
\hline $\begin{array}{l}\text { Área Contaminada sob } \\
\text { Intervenção }(\mathrm{ACl})\end{array}$ & $\begin{array}{l}\text { Terreno onde foi constatada a presença de hidrocarbonetos em fase livre ou foi comprovada, } \\
\text { após IPA Detalhada e Avaliação de Risco, a existência de risco à saúde humana e ao meio } \\
\text { ambiente. Áreas em processo de remediação também foram enquadradas nesta categoria. }\end{array}$ \\
\hline $\begin{array}{l}\text { Área em Processo de } \\
\text { Monitoramento } \\
\text { Reabilitação (AMR) }\end{array}$ & sco foi considerado tolerável após a Avaliação de Risco. \\
\hline $\begin{array}{l}\text { Área Livre de Contaminação } \\
\text { (ALC) }\end{array}$ & $\begin{array}{l}\text { Aquela onde, após a IPA Preliminar, não foram observados indícios de contaminação. Esta } \\
\text { categoria foi estabelecida pelos autores, pois não consta dentre as definidas pelo pela Resolução } \\
\text { no 420/2009, do CONAMA. }\end{array}$ \\
\hline
\end{tabular}

Com base nos dados obtidos neste estudo, realizou-se o enquadramento de cada um dos terrenos pesquisados em uma das cinco categorias descritas no Quadro 1, estabelecidas por Brasil (2009). Com o auxílio do software ArcGis 10.2, foram elaborados mapas temáticos para espacialização das informações inventariadas, o que facilitou a análise e a discussão dos resultados, inclusive frente à experiência internacional e nacional no gerenciamento de áreas contaminadas.

\section{RESULTADOS E DISCUSSÃO}

\section{Enquadramento quanto aos níveis de contaminação ambiental}

Dentre os 200 postos de serviços do município de Goiânia/GO contemplados na pesquisa, 44 (22\%) foram enquadrados como Áreas Suspeitas de Contaminação, aquelas em que, após a realização de IPA 
Preliminar, foram identificados indícios da presença de contaminação ou condições que possam representar perigo; e 23 (11,5\%) como Áreas Contaminadas sob Investigação, onde comprovadamente foi constatada a presença de hidrocarbonetos (BTEXs e hidrocarbonetos policíclicos aromáticos - PAHs) no solo e/ou nas águas subterrâneas acima dos valores máximos estabelecidos por Brasil (2009). As Áreas Contaminadas sob Intervenção, formadas por terrenos onde há comprovação da existência de riscos à saúde humana, em processo de remediação ou com presença de contaminação do tipo fase livre, somaram 15 (7,5\%). As Áreas em Processo de Monitoramento para Reabilitação, onde o risco foi considerado tolerável após a Avaliação de Risco, perfizeram o total de 3 (1,5\%), enquanto as Áreas Livres de Contaminação somaram 115 (57,5\%) (Figura 2).

Apesar de pouco numerosas se comparadas às demais categorias, as Áreas Contaminadas sob Intervenção, principalmente se somadas às Áreas Contaminadas sob Investigação, com presença comprovada de hidrocarbonetos, e às Áreas Suspeitas de Contaminação, que após o aprofundamento das investigações de passivo ambiental poderão ser enquadradas como contaminadas, representam o cenário ambientalmente mais crítico identificado neste estudo. Tendo em vista o universo amostral de 200 processos, o total de 82 terrenos (41\%) com presença comprovada de hidrocarbonetos ou com suspeita de contaminação é um número elevado para uma única cidade, representando um preocupante desafio para os gestores públicos de Goiânia, principalmente devido ao fato de que muitos desses terrenos apresentam contaminações ambientalmente complexas, ou seja, de difícil resolução.

Segundo Mariano et al. (2017) e Valentine et al. (2018), a configuração de cenários de contaminação por derivados de petróleo e seus graus de complexidade ambiental associados variam em função de diversos fatores não controlados, como tipo de solo, declividade, clima, profundidade do nível piezométrico, sentido de escoamento das águas subterrâneas e taxas de biodegradação, difusão e de dispersão das plumas. Todavia, mesmo quando o site apresenta hidrocarbonetos em baixas concentrações, é possível que organismos expostos venham a desenvolver enfermidades graves. Nesse sentido, Yuan et al. (2014) e Monaco et al. (2015) destacam que, à medida que as áreas contaminadas apresentam maior complexidade devido ao comprometimento de mais de um recurso natural, ou que as plumas alcançam distâncias e receptores de risco localizados além dos limites físicos dos terrenos onde os passivos ambientais se originaram, a situação gerencial tende a se tornar mais desafiadora, dificultando a atuação do poder público.

De acordo com Ite et al. (2018), o gerenciamento de passivos ambientais de postos de serviços pode ser agravado se ocorrer o uso intencional ou desavisado de terrenos contaminados para fins habitacionais, dada a elevação do risco de exposição humana. No estudo desenvolvido por Cortês et al. (2011), por exemplo, uma área de aproximadamente 180 mil $\mathrm{m}^{2}$ localizada no bairro de Vila Carioca, cidade de São Paulo, e contaminada com BTEXs, desencadeou sérios problemas ambientais e sociais devido à magnitude da contaminação. Por sua vez, Silva et al. (2002), ao pesquisarem a contaminação provocada por um posto de combustível situado em Itaguaí (RJ), constataram que dois anos após a ocorrência de um grande vazamento, em cerca de $20 \%$ dos poços rasos das residências do entorno foram detectadas concentrações de benzeno 100 vezes maiores que o valor máximo permitido para as coleções hídricas subterrâneas, demonstrando que 
as plumas haviam extravasado os limites do terreno onde a contaminação se originou. Segundo Vasconcelos et al. (2014) e Lima et al. (2017), um dos principais fatores capazes de acelerar e ampliar o alcance subterrâneo de plumas de hidrocarbonetos é a presença de etanol na gasolina, cuja concentração de pelo menos $10 \%$ já é capaz de alterar significativamente a solubilidade dos compostos químicos de interesse. No caso brasileiro, a composição de etanol na gasolina é em torno de $25 \%$.

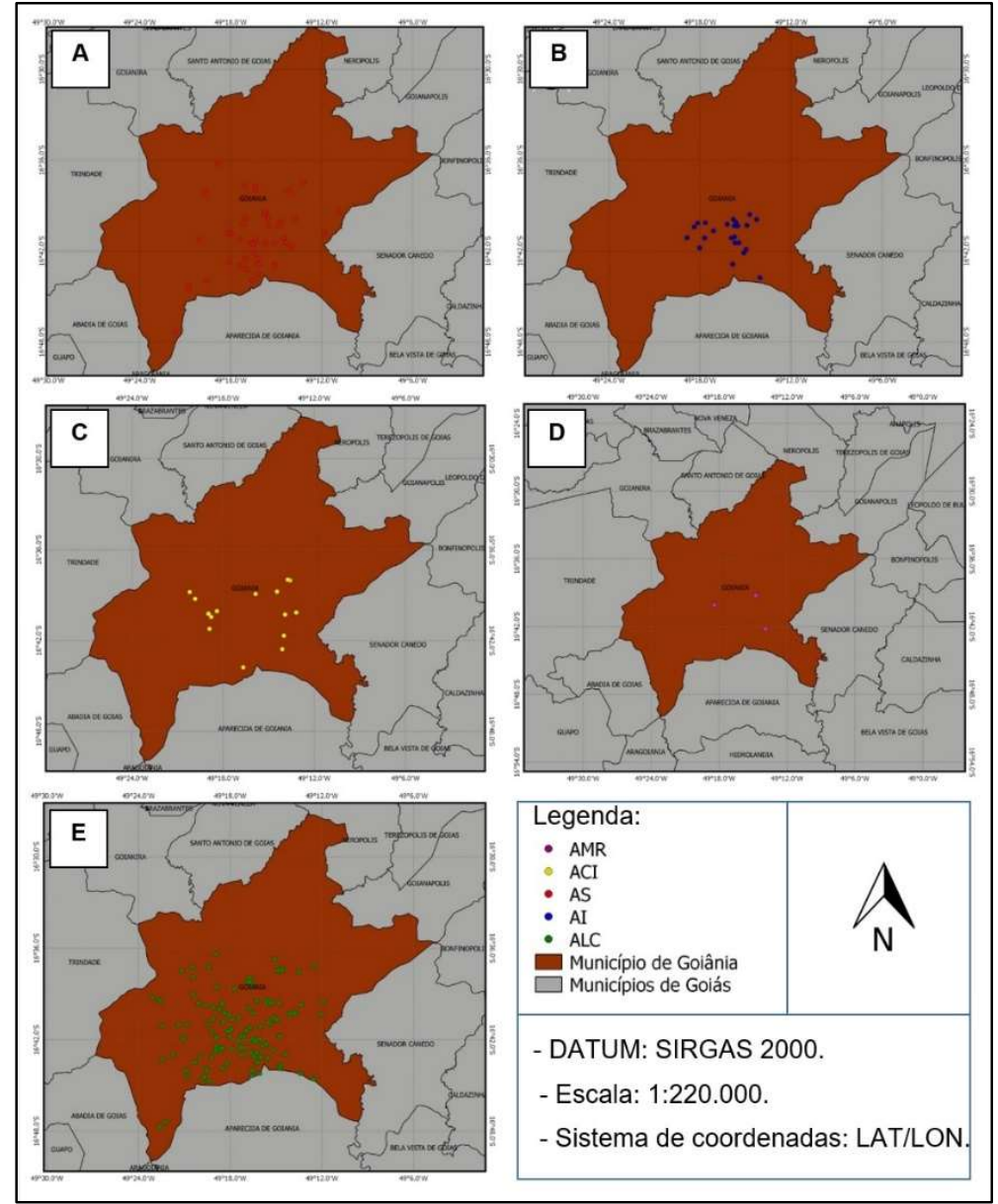

Figura 2: Áreas de Goiânia/GO classificadas como a) Suspeitas de Contaminação; b) Contaminadas sob Investigação; c) Contaminadas sob Intervenção; d) Em Processo de Monitoramento para Reabilitação e e) Livres de Contaminação.

Além dos desafios associados ao gerenciamento de cada passivo ambiental propriamente dito, o número de áreas contaminadas por combustíveis no Brasil tem se elevado nos últimos anos, dado o crescente aumento do uso de combustíveis. Apenas em São Paulo, por exemplo, haviam 255 terrenos contaminados por derivados de petróleo em maio/2002, enquanto que em 2010 esse número subiu para 2922, um aumento de mais de 1100\% (MORAES et al., 2014). Estes mesmos autores complementam que, de 2004 a 2007, o Ministério da Saúde (MS) desenvolveu um estudo de análise de 700 áreas contaminadas no país, sendo que das 128 existentes na Região Sudeste, a maioria foi desencadeada por postos de serviços. Goiânia, por se tratar de uma capital cada vez mais urbanizada e com número crescente de postos, também tende a apresentar elevação temporal do número de áreas contaminadas por hidrocarbonetos.

Na Figura 3 é apresentada a sobreposição dos resultados obtidos para todas as categorias de enquadramento utilizadas, onde se observa a formação de quatro grandes aglomerados de terrenos (Áreas 1, 2, 3 e 4) localizados na parte central do núcleo urbano que, devido às suas condições ambientais, 
demonstram ser prioritários para fins de gerenciamento, pois englobam conjuntos de empreendimentos classificados como Áreas Contaminadas sob Intervenção, Áreas Contaminadas sob Investigação e Áreas Suspeitas de Contaminação. Especificamente na Área 1, compreendida por bairros como Centro, Castelo Branco, Sol Nascente, Jardim Guanabara, Cidade Jardim, Rodoviário e Aeroviário, o poder público local necessitará intensificar os esforços de fiscalização e de monitoramento, devido ao fato de serem setores com maiores números de pessoas potencialmente expostas a riscos como inalação de vapores. Por outro lado, nas Áreas 2, 3 e 4, constituídas de bairros mais periféricos, embora o número de cidadãos potencialmente expostos seja menor, o uso das águas subterrâneas é mais intenso, especialmente por parte da população de baixa renda, resultando na elevação dos riscos associados à exposição via ingestão e ao contato dermal com água e/ou solos contaminados.

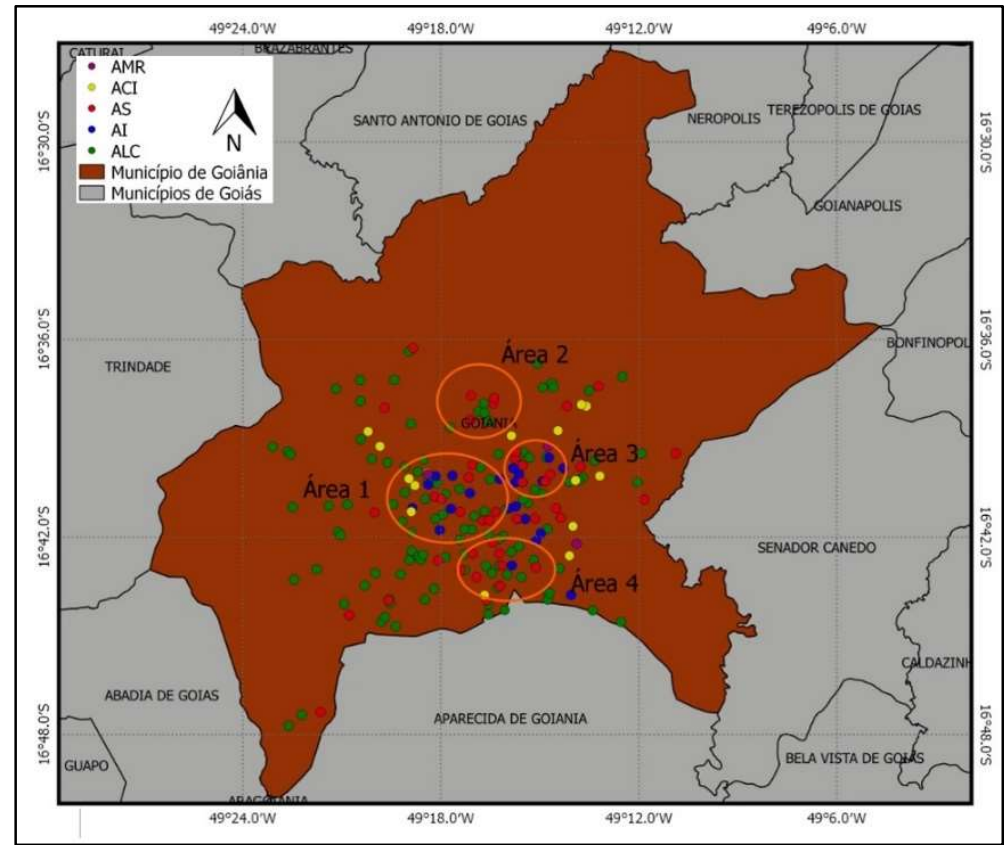

Figura 3: Sobreposição das áreas localizadas em Goiânia/GO, classificadas em uma das cinco categorias de enquadramento estabelecidas no estudo, incluindo a indicação das regiões demandantes de ações prioritárias de gerenciamento por parte da municipalidade.

Verifica-se (Figura 3) que a realidade das áreas contaminadas por hidrocarbonetos em Goiânia se mostra ambientalmente preocupante, dado o número de terrenos ambientalmente problemáticos que demandarão ações prioritárias de fiscalização e monitoramento oriundas de planos resolutivos estratégicos. Resultado semelhante foi obtido no levantamento realizado por MPRN (2016), desenvolvido nos 110 postos de serviços de Natal (RN). No trabalho, após vistorias realizadas com o objetivo de inspecionar instalações e checar se existiam indícios de contaminação, foi constatado que 109 estabelecimentos apresentavam algum tipo de vazamento no SASC, realidade que se não descoberta mediante fiscalização preventiva, certamente teria resultado na intensificação dos passivos ambientais naquele município. Nesse sentido, os postos de serviços, hidrocarbonetos podem se originar de derramamentos acidentais durante as operações de descarga, erros operacionais e danos nos SASCs. Especificamente, tanques costumam apresentar vida útil média de vinte anos, dada a propensão estatística de ocorrência de problemas de estanqueidade, o que tem movimentado o setor a paulatinamente substituir essas unidades armazenadoras simples por outras de 
parede dupla e dotadas de monitoramento intersticial (DIAS, 2012).

\section{A experiência internacional e nacional no gerenciamento de áreas contaminadas e suas possíveis contribuições para a solução dos problemas identificados em Goiânia}

Internacionalmente, o gerenciamento de passivos ambientais vem sendo realizado por gestores públicos municipais, efetivos conhecedores das realidades ambientais locais. De acordo com Sousa (2001), à medida que as esferas mais altas dos governos se tornam mais conscientes dos custos e riscos que devem compartilhar para efetivamente resolverem o problema das áreas contaminadas, as políticas e programas de gerenciamento tendem a convergir, em estilo e conteúdo, para a valorização da gestão local, fato observado na Europa e Canadá. Luo et al. (2009) desenvolverem um estudo comparativo dos programas de gerenciamento de áreas contaminadas existentes na China e no Reino Unido, tendo concluído que as providências adotadas deveriam ser protagonizadas pela municipalidade e precisariam estar focadas, prioritariamente, nos terrenos problemáticos, sendo necessário o desenvolvimento de abordagens técnicas baseadas no risco e de um sistema integrado de gestão que incluísse incentivos financeiros associados a um planejamento detalhado.

Estudos mais recentes também apontam para a importância do protagonismo da administração local no que se refere ao gerenciamento de áreas contaminadas por postos de serviços. Ao estudarem cenários de contaminação em Stuttgart, uma cidade alemã altamente industrializada e com mais de 500 áreas oficialmente declaradas como contaminadas, Vasin et al. (2016) concluíram que as práticas de gestão ambiental aplicadas e as medidas para mitigação das fontes de poluição por combustíveis não foram suficientes, sendo necessários ajustes seguidos de monitoramentos por parte do poder público local. Por sua vez, Tarafdar et al. (2018), ao pesquisarem terrenos de deposição de lodo de uma refinaria de petróleo na Índia, comprovaram que as populações que viviam nas proximidades dos locais contaminados por PAHs estavam sujeitas a maiores riscos de desenvolvimento de câncer, cabendo ao governo local a adoção de ações imediatas de gerenciamento.

Oughton et al. (2004) defendem que as estratégias de recuperação de áreas contaminadas precisam considerar uma ampla gama de diferentes questões para garantirem a sustentabilidade a longo prazo, não devendo abrir mão da viabilidade sócio-econômica-ambiental, e de aspectos como percepção pública de risco, comunicação de informação, necessidade de diálogo e consulta com as comunidades afetadas. Nesse sentido, em seu estudo comparativo das medidas adotadas para fins de gerenciamento de áreas contaminadas em 31 regiões da China, Li et al. (2017) destacaram que, ao contrário dos EUA, Canadá, Japão e Europa, que têm experiências ricas acumuladas sobre o tema, a China ainda carece de estratégias eficazes de ações resolutivas dos seus passivos ambientais, assim como o Brasil. Nesse sentido, os pesquisadores concluíram que medidas voltadas à solução do problema apresentavam semelhanças e diferenças dentro do próprio território chinês, sendo necessária a implementação de um sistema objetivo de vários níveis combinado com diversos instrumentos fundamentados na cooperação entre as diferentes partes interessadas, incluindo, ainda, um mecanismo de supervisão conjunta. Adicionalmente, enfatizaram a 
necessidade de instituição de políticas especializadas, divulgação de informações à população exposta, participação do público nas tomadas de decisão e adoção de ações corretivas emergenciais, medidas que deverão ser fortalecidas pela gestão local.

No caso do Brasil, o gerenciamento de áreas contaminadas por hidrocarbonetos na maior parte das Unidades Federativas é prerrogativa exclusiva do poder estadual, embora em Goiás o Governo venha estimulando a descentralização do licenciamento e conferindo essa responsabilidade aos municípios, como já acontece em Goiânia. Todavia, Sánchez (2001) destaca que, independente do ente público designado para atuar, as decisões gerenciais necessitam estar embasadas em diagnósticos ambientais tecnicamente consistentes, incluindo o solo e o subsolo, com todos seus componentes sólidos, líquidos e gasosos. Para o sucesso do gerenciamento, o autor também salienta a importância da existência de estruturas física e humana satisfatórias. Nesse sentido, contudo, a maior parte dos órgãos de meio ambiente municipais brasileiros ainda são despreparados frente às especificidades afetas ao licenciamento de postos de serviços, realidade que impede a obtenção de bons resultados gerenciais e que, muitas vezes, resulta no agravamento dos cenários de contaminação, administrados de maneira inadequada ou simplesmente negligenciados pelo poder público por falta de conhecimento técnico. Diante dessa realidade, a atuação do poder público muitas vezes ainda ocorre em caráter emergencial, quando a contaminação já está instalada, e não de maneira preventiva, resultando em maiores prejuízos ao meio ambiente, aos empreendedores e à sociedade como um todo (SEPE et al., 2004).

Especificamente no caso de Goiânia, embora o gerenciamento de passivos ambientais de postos de combustíveis já seja realizado pela administração pública municipal há anos, os resultados obtidos com o presente estudo demonstram a necessidade de seu avanço, tendo em vista a elevada quantidade de Áreas Contaminadas sob Intervenção, Áreas Contaminadas sob Investigação e Áreas Suspeitas de Contaminação a serem administradas. Nesse sentido, embora em muitos casos as medidas gerenciais necessárias se mostrem desafiadoras, independente do grau de complexidade que os sites apresentem, as decisões administrativas necessitam estar embasadas em critérios que possibilitem a obtenção de resultados satisfatórios a curto, médio e longo prazo, sob pena de se ter pessoas e o meio ambiente submetidos a riscos desnecessários. Para tanto, intervenções específicas precisam ser implementadas de maneira ordenada, desde o momento do diagnóstico propriamente dito até o monitoramento das ações executadas, uma responsabilidade a ser compartilhada pelo poder público local e pelos proprietários dos postos, desenvolvida em plena consonância com os princípios e objetivos estabelecidos por Brasil (2009).

Naturalmente, o órgão ambiental de Goiânia precisará aprimorar a implementação de ações de controle das áreas contaminadas por postos de serviços, sendo que as alternativas de intervenção definidas poderão contemplar, de forma não excludente, a eliminação do perigo ou a redução a níveis toleráveis dos riscos à saúde humana e ao meio ambiente; o zoneamento e a restrição dos usos e ocupação do solo e das águas superficiais e subterrâneas; a aplicação de técnicas de remediação e o monitoramento ambiental (BRASIL, 2009). Para tanto, deverão ser implementadas ações emergenciais em casos de identificação de condições de perigo; avaliações detalhadas dos diagnósticos ambientais apresentados; comunicações de 
risco após a declaração das áreas como Contaminadas Sob Intervenção; avaliações das propostas de intervenção dos terrenos; acompanhamento das ações emergenciais, de intervenção e de monitoramento; avaliações da eficácia das ações de intervenção e, finalmente, a publicação e a comunicação da situação da área ao proprietário, ao possuidor, ao Cartório de Registro de Imóveis e ao cadastro imobiliário da Prefeitura de Goiânia, de forma a registrar a situação ambiental dos terrenos degradados e/ou em processo de recuperação (BRASIL, 2009).

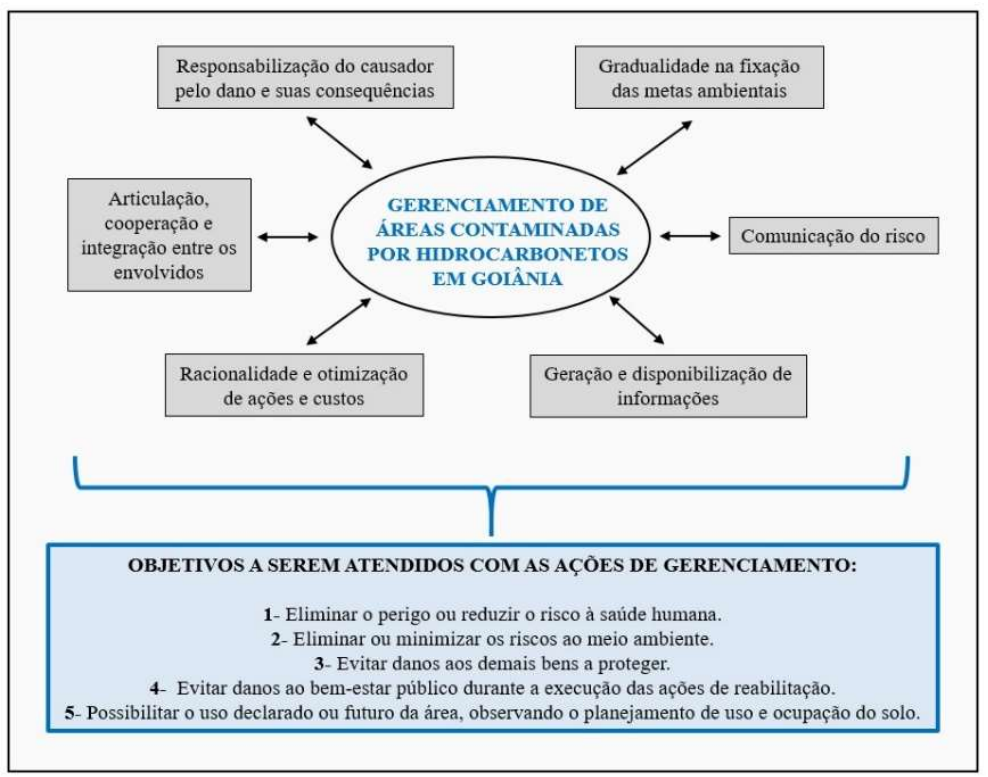

Figura 4: Princípios e objetivos estabelecidos por Brasil (2009), a serem atendidos no gerenciamento das áreas contaminadas por hidrocarbonetos identificadas em Goiânia/GO.

Também é fundamental que as medidas de gerenciamento de áreas contaminadas adotadas sejam embasadas em ações que assegurem o conhecimento da qualidade das águas subterrâneas e do solo afetado, proporcionando a geração das informações mínimas necessárias à intervenção correta no âmbito gerencial. Do contrário, os terrenos atingidos poderão passar a atuar como fontes dinâmicas de contaminação secundária, cujos efeitos tenderão a ultrapassar os limites das áreas afetadas, aumentando a complexidade do problema e expondo outros recursos naturais e humanos a riscos originalmente não controlados. Por outro lado, os responsáveis pelos postos de serviços precisarão continuar mantendo a submissão dos estudos técnicos específicos voltados ao gerenciamento de áreas contaminadas por hidrocarbonetos à Agência Municipal do Meio Ambiente de Goiânia, principalmente das propostas de intervenção a serem executadas, devendo estas considerarem o controle ou a eliminação das fontes de contaminação; o uso atual e futuro do solo da área objeto e sua circunvizinhança; a avaliação de risco à saúde humana; as alternativas de intervenção consideradas técnica e economicamente viáveis e suas consequências; o programa de monitoramento da eficácia das ações executadas e os custos e prazos envolvidos na implementação das alternativas de intervenção propostas para atingir as metas estabelecidas. Adicionalmente, deve ser informado o uso pretendido dos terrenos contaminados, cabendo ao órgão licenciador decidir sobre sua viabilidade ambiental com fundamento na legislação vigente, no diagnóstico da área, na avaliação de risco, nas ações de intervenção propostas e no zoneamento do uso do solo (GUNTHER, 2006; BRASIL, 2009). 


\section{CONCLUSÕES}

Embora águas subterrâneas e solos contaminados por hidrocarbonetos apresentem elevado potencial de degradação do meio natural e de toxicidade aos seres vivos, são um problema ambiental ainda pouco estudado no Brasil. Nesse sentido, o presente trabalho objetivou apresentar um panorama das áreas contaminadas por hidrocarbonetos oriundos de postos de serviços localizados em Goiânia, Goiás, Brasil, visando contribuir para o avanço das discussões associadas ao tema. Especificamente, os resultados obtidos possibilitaram identificar quatro aglomerados de áreas contaminadas demandantes de atenção prioritária em termos de gestão ambiental preventiva e corretiva - por parte do poder público local, o que poderá ser alcançado mediante a melhoria das ações de fiscalização e responsabilização, dos instrumentos de controle para recuperação e das medidas de acompanhamento dos recursos humanos e ambientais atingidos ou expostos. Naturalmente, para que se consiga alcançar resultados gerenciais satisfatórios, é fundamental um maior envolvimento de outros atores sociais, como o setor privado e a sociedade civil.

Considerando a contínua expansão da rede de postos de combustíveis nas cidades brasileiras, associada aos desafios de gestão dos passivos ambientais gerados pelo setor, fica clara a crescente necessidade de aplicação de medidas gerenciais sistêmicas locais voltadas à proteção dos recursos naturais e humanos expostos, bem como à reintegração dos terrenos impactados ao tecido urbano, na maior proximidade possível das suas condições originais. Portanto, o tema precisa ser considerado como componente prioritário na busca pela sustentabilidade do município de Goiânia, devendo, inclusive, figurar em políticas públicas preventivas e corretivas específicas, até então incipientes em praticamente todo o território nacional.

\section{REFERÊNCIAS}

ADIPAH, S.. Introduction of petroleum hydrocarbons contaminants and its human effects. Journal of Environmental Science and Public Health, v.3, n.1, p.001009, 2019. DOI: http://doi.org/10.26502/jesph.96120043

ARAÚJO-MOURA, A. A. C.; CAFFARO FILHO, R. A.. Panorama do gerenciamento de áreas contaminadas no Brasil após a Resolução CONAMA 420/09. Águas Subterrâneas, v.29, n. 2, p.202-212, 2015. DOI:

http://doi.org/10.14295/ras.v29i2.27972

BALKIS, N.; AKSU, A.; ERSAN, M.. Petroleum hydrocarbon contamination of the Southern Black Sea Self, Turkey. Environmental Science and Pollution Research, v.19, n.2, p.592-599, 2012. DOI: http://doi.org/10.1007/s11356-011$\underline{0583-4}$

BALSEIRO-ROMERO, M.; MACÍAS, F.; MONTERROSO, C.. Characterization and fingerprinting of soil and groundwater contamination sources around a fuel distribution station in Galicia (NW Spain). Environmental Monitoring and Assessment, v.188, n.5, 2016. DOI: http://doi.org/10.1007/s10661-016-5288-1

BOCOS, E.; FERNANDEZ-COSTAS, C.; PAZOS, M.; SANROMAN, A. M.. Removal of PAHs and pesticides from polluted soils by enhanced electrokinetic-Fenton treatment. Chemosphere, v.125, p.168-174, 2015. DOI:

http://doi.org/10.1016/j.chemosphere.2014.12.049

BRASIL. Resolução n. 273, de 29 de novembro de 2000. Estabelece diretrizes para o licenciamento ambiental de postos de combustíveis e serviços e dispõe sobre a prevenção e controle da poluição. Brasília: DOU, 2001. p.2023.

BRASIL. Resolução n. 420, de 28 de dezembro de 2009. Dispõe sobre critérios e valores orientadores de qualidade do solo quanto à presença de substâncias químicas e estabelece diretrizes para o gerenciamento ambiental de áreas contaminadas por essas substâncias em decorrência de atividades antrópicas. Brasília: DOU, 2009. p.81-84.

BROWN, P. J.; LONG, S. M.; SPURGEON, D. J.; SVENDSEN, C.; KANKARD, P. K.. Toxicological and biochemical responses of the earthworm Lumbricus rubellus to pyrene, a noncarcinogenic polycyclic aromatic hydrocarbon. Chemosphere, v.57, n.11, p.1675-1681, 2004. DOI: http://doi.org/10.1016/j.chemosphere.2004.05.041

CAMENZULI, D.; FREIDMAN, B. L.. On-site and in situ remediation technologies applicable to petroleum hydrocarbon contaminated sites in the Antarctic and Arctic. 
Polar Research, v.34, n.1, p.1-19, 2015. DOI: http://doi.org/10.3402/polar.v34.24492

CHEN, C. W.; CHEN, C. F.. Distribution, origin, and potencial toxicological significance of polycyclic aromatic hydrocarbons (PAHs) in sediments of Kaohsiung Harbor, Taiwan. Marine Polution Bulletin, v.63, n.5-12, p.417-423, 2011. DOI: http://doi.org/10.1016/j.marpolbul.2011.04.047

CETESB. Companhia de Tecnologia de Saneamento Ambiental do Estado de São Paulo. Decisão de Diretoria n. 038/2017/C. São Paulo: CETESB, 2017.

CORTÊS, P. L.; FILHO, A. P. A.; RUIZ, M. S.; TEIXEIRA, C. E.. A deposição de resíduos industriais organoclorados no litoral do Estado de São Paulo: um estudo de caso. Revista de Administração e Inovação, v.8, n.2, p.131-163, 2011. DOI: http://doi.org/10.5773/rai.v8i2.700

\section{DIAS, G. M. Adequação ambiental dos postos de} combustíveis de Natal e recuperação da área degradada. Natal: MPRN, 2012.

DJOMO, J. E.; DAUTA, A.; FERRIER, V.; NARBONNE, J. F.; MONKIEDJE, A.; NJINE, T.; GARRIGUES, P.. Toxic effects of some major polyaromatic hydrocarbons foun in crude oil and aquatic sediments on Scenedesmus subspicatus. Water Research, v.38, n.7, p.1817-1821, 2004. DOI: http://doi.org/10.1016/i.watres.2003.10.023

FORTE, E. J.; AZEVEDO, M. S.; OLIVEIRA, R. C.; ALMEIDA, R.. Contaminação de aquífero por hidrocarbonetos: estudo de caso na Vila Tupi, Porto Velho, Rondônia. Química Nova, v.30, n.7, p.1539-1544, 2007. DOI:

http://doi.org/10.1590/S0100-40422007000700008

FREIRE, P. A. C.; TRANNIN, I. C. B.; SIMÕES, S. J.. Bombeamento e tratamento da fase livre em aquífero litorâneo. Engenharia Sanitária e Ambiental, v.19, n.4, p.461-470, 2014. DOI: http://doi.org/10.1590/S141341522014019000000122

GŪNTHER, W. M. R.. Áreas contaminadas no contexto da gestão urbana. São Paulo em Perspectiva, v.20, n.2, p.105117, 2006.

HILPERT, M.; MORA, B.A.; NI, J.; RULE, A. M.; NACHMAN, K. E.. Hydrocarbon release during fuel storage and transfer at gas stations: environmental and health effects. Current Environmental Health Reports, v.2, n.4, p.412-422, 2015. DOI: https://doi.org/101007/s40572-015-0074-8

IBGE. Instituto Brasileiro e Geografia E Estatística. Perfil dos municípios brasileiros. Rio de Janeiro: IBGE, 2016.

ISTRATE, I. A.; COCÂRTÅ, D. M.; WU, Z.; STOIAN, M. A.. Minimizing the health risks from hydrocarbon contaminated soils by using Electric Field-Based Treatment for soil remediation. Sustainability, n.10, n.1, p.1-13, 2018. DOI: https://doi.org/10.3390/su10010253

ITE, A. E.; HARRY, T. A.; OBADIMU, C. O.; ASUAIKO, E. R.; INIM, I. J.. Petroleum hydrocarbons contamination of surface water and groundwater in the Niger Delta Region of Nigeria. Journal of Environment Pollution and Human Health, v.6, n.2, p.51-61, 2018. DOI: http://doi.org/10.12691/jephh-6-2$\underline{2}$
KHAN, A. A.; COPPOCK, R. W.; SCHULER, M. M.; FLORENCE, L. Z.; LILLIE, L. E.; MOSTROM, M. S.. Biochemical effects of pembina cardium crude oil exposure in Cattle. Archives of Environmental Contamination and Toxicology, v.30, p.349355, 1996. DOI: http://doi.org/10.1007/BF00212293

LI, X.; JIAO, W.; XIAO, R.; CHEN, W.; LIU, W.. Contaminated sites in China: countermeasures of provincial governments. Journal of Cleaner Production, v.147, p.485-496, 2017. DOI: http://doi.org/10.1016/j.jclepro.2017.01.107

LIMA, S. D.; OLIVEIRA, A. F.; GOLIN, R.; CAIXETA, D. S.; LIMA, Z. M.; MORAIS, E. B.. Gerenciamento de áreas contaminadas por postos de combustíveis em Cuiabá, Mato Grosso, Brasil. Revista Ambiente \& Água, v.12, n.2, p.300-315, 2017. DOI: http://doi.org/10.4136/ambi-agua.1872

LUO, Q.; CATNEY, P.; LERNER, D.. Risk-based management of contaminated land in the UK: lessons for China? Journal of Environmental Management, v.90, n.2, p.112-125, 2009. DOI: http://doi.org/10.1016/i.jenvman.2008.05.001

MARIANO, A. P.; ANGELIS, D. F.; BONOTTO, D. M.. Monitoramento de indicadores geoquímicos e avaliação de biodegradação em área contaminada com óleo diesel. Engenharia Sanitária e Ambiental, v.12, n.3, p.296-304, 2017. DOI: http://dx.doi.org/101590/S141341522007000300009

MONACO, D.; RICCIO, A.; CHIANESE, E.; ADAMO, P.; ROSA, S.; FAGNANO, M.. Chemical characterization and spatial distribution of PAHs and heavy hydrocarbons in rural sites of Campania Region, South Italy. Environmental Sicience and Pollution Research, v.22, n. 19, p.14993-15003, 2015. DOI: http://dx.doi.org/10.1007/s11356-015-4733-y

MORAES, S. L.; TEIXEIRA, C. E.; MAXIMIANO, A. M. S.. Guia de elaboração de planos de intervenção para o gerenciamento de áreas contaminadas. São Paulo: IPT, 2014.

OUGHTON, D.; FORSBERG, E.; BAY, I.; KAISER, M.; HOWARD, $B$.. An ethical dimension to sustainable restoration and longterm management of contaminated areas. Journal of Environmental Radioactivity, v.74, n.1-3, p.171-183, 2004. DOI: http://dx.doi.org/10.1016/j.jenvrad.2004.01.009

RODRÍGUEZ, C.; IGLESIAS, K.; BÍCEGO, M. C.; TANIGUCHI, S. Hydrocarbons in soil and meltwater stream sediments near Artigas Antarctic Research Station: origin, sources and levels. Antartic Science, v.30, n.3, p.170-182, 2018. DOI: http://dx.doi.org/10.1017/S0954102018000019

ROSALES, R. M.; MARTÍNEZ-PAGÁN, P.; FAZ, A.; BECH, J. Study of subsoil in former petrol stations in SE of Spain: physicochemical characterization and hydrocarbon contamination assessment. Journal of Geochemical Exploration, v.147, p.306-320, 2014. DOI: http://doi.org/10.1016/j.gexplo.2014.10.006

SAM, K.; COULON, F.; PRPICH, G.. Management of petroleum hydrocarbon contaminated sites in Nigeria: current challenges and future direction. Land Use Policy, v.64, p.133-144, 2017. DOI: http://doi.org/10.1016/j.landusepol.2017.01.051 
SÁNCHEZ, L. E.. Desengenharia: o passivo ambiental na desativação de empreendimentos Industriais. São Paulo: USP, 2001.

SEPE, P. M.; SILVA, F. A. N.. Remediação e revitalização de contaminadas: aspectos técnicos, legais e financeiros. São Paulo: SIGNUS, 2004

SILVA, R. L. B.; BARRA, C. M.; MONTEIRO, T. C. N.; BRILHANTE, O. M.. Estudo da contaminação de poços rasos por combustíveis orgânicos e possíveis consequências para a saúde pública no município de Itaguaí, Rio de Janeiro, Brasil. Caderno de Saúde Pública, v.18, p.1599-1607, 2002. DOI: http://doi.org/10.1590/S0102-311X2002000600014

SINGH, K.; CHANDRA, S.. Treatment of petroleum hydrocarbon polluted environment through bioremediation: a review. Pakistan Journal of Biological Sciences, v.17, n.1, p.1-8, 2014. DOI: http://doi.org/10.3923/pjbs.2014.1.8

SOUSA, C.. Contaminated sites: the Canadian situation in an international context. Journal of Environmental Management, v.62, n.2, p.131-154, 2001. DOI: https://doi.org/10.1006/jema.2001.0431

SOUZA, E. C.; VESSONI-PENNA, T. C.; OLIVEIRA, R. P. S. Biosurfactant-enhanced systems contaminated with petroleum hydrocarbons and ethanol. Water Research, v.32, p.654-660, 2014.

SGM. Superintendência de Geologia e Mineração de Goiás. Mapa geológico: relatório final. Goiânia: Secretaria da Indústria e Comércio, 2005.

TARAFDAR, A.; SINHA, A.. Public health risk assessment with bioaccessibility considerations for soil PAHs at oil refinery vicinity areas in India. Science of the Total Environment, v.616-617, p.1477-1484, 2018. DOI: http://dx.doi.org/10.1016/j.scitotenv.2017.10.166
TEIXEIRA, C. E.; MOTTA, F. G.; MORAES, S. L.. Panorama GAC: mapeamento da cadeia de gerenciamento de áreas contaminadas. São Paulo: IPT, 2016.

UGOCHUKWU, U. C.; OCHONOGOR, A.; JIDERE, C. M.; AGU, C.; NKOLOAGU, F.; EWOH, J.; OKWU-DELUNZU, V. U.. Exposure risck to polycyclic aromatic hydrocarbons by humans and livestock (cattle) due to hydrocarbon spill from petroleum products in Niger-delta wetland. Environment International, v.115, p.38-47, 2018. DOI: http://dx.doi.org/10.1016/j.envint.2018.03.01

VALENTYNE, A.; CRAWFORD, K.; COOK, T.; MATHEWSON, P. D.. Polycyclic aromatic hydrocarbon contamination and source profiling in watersheds serving three small Wisconsin, USA cities. Science of the Total Environment, v.627, p.14531463, 2018. DOI: http://doi.org/10.1016/i.scitotenv.2018.01.200

VASCONCELOS, B. D.; SANTOS, M. L. L. M.; VILELA, M. M. O. P.; CARVALHO, T. D.; FONSECA, F. L. A.; SILVA, O. R.; JUNQUEIRA, V. B. C.; AZZALIS, L. A.; SOLDÁ, P. L.. Áreas contaminadas por postos de combustível e medidas de remediação no município de São Bernardo do Campo. Revista Saúde e Meio Ambiente, v.3, p.73-83, 2014. DOI: http://doi.org/10.24302/sma.v3i1.539

VASIN, S.; ACHIM, C.; ULRICHM L.; HERMANN JOSEF, K.. A groundwater management plan for Stuttgart. Science of the Total Environment, v.563-564, p.704-712, 2016. DOI: http://doi.org/10.1016/j.scitotenv.2015.10.078

YUAN, S.; LI, K.; CHEN, T.; BI, X.; WANG, Q. Y.. Soil contamination by polycyclic aromatic hydrocarbons at natural recreational areas in Delaware, USA. Environmental Earth Sciences, v.72, n.2, p.387-398, 2014. DOI: http://doi.org/10.1007/s12665-013-2959-x

ZELINKOVA, Z.; WENZL, T.. The occurrence of 16 EPA PAHs in food: a review. Polyclyc Aromat Compd, v.35, p.248-284, 2015. DOI: http://doi.org/10.1080/10406638.2014.918550

A CBPC - Companhia Brasileira de Produção Científica (CNPJ: 11.221.422/0001-03) detém os direitos materiais desta publicação. Os direitos referem-se à publicação do trabalho em qualquer parte do mundo, incluindo os direitos às renovações, expansões e disseminações da contribuição, bem como outros direitos subsidiários. Todos os trabalhos publicados eletronicamente poderão posteriormente ser publicados em coletâneas impressas sob coordenação da Sustenere Publishing, da Companhia Brasileira de Produção Científica e seus parceiros autorizados. Os (as) autores (as) preservam os direitos autorais, mas não têm permissão para a publicação da contribuição em outro meio, impresso ou digital, em português ou em tradução. 